\title{
ECG signal denoising using discrete wavelet transform: A comparative analysis of threshold values and functions
}

\author{
Marco V. Gualsaquí M., Iván P. Vizcaíno E., Víctor G. Proaño R., Marco J. Flores C (D. \\ Departamento de Eléctrica y Electrónica de la Universidad de la Fuerzas Armadas ESPE, Av. Gral. \\ Rumiñahui, s/n, PBX 171-5-231B, Sangolquí (Pichincha), Ecuador.
}

Autores para correspondencia: mvgualsaqui@espe.edu.ec, mjflores@espe.edu.ec

Fecha de recepción: 5 de abril 2018 - Fecha de aceptación: 18 de junio 2018

\begin{abstract}
The electrocardiogram signal $(E C G)$ is a bio-signal used to determine cardiac health. However, different types of noise that commonly accompany these signals can hide valuable information for diagnosing disorders. The paper presents an experimental study to remove the noise in $E C G$ signals using the Discrete Wavelet Transform $(D W T)$ theory and a set of thresholds filters for efficient noise filtering. For the assessment process, we used ECG records from MIT-BIH Arrhythmia database (MITDB) and standardized noise signals (muscle activity and electrode-skin contact) database from the Noise Stress Test database. In addition to the ECG signals a white Gaussian noise present in electrical type signals was added. Furthermore, as a first step we considered baseline wander and power line interference reduction. The metrics used are the Signal-to-Noise Ratio $(S N R)$, the Root Mean Squared Error (RMSE), the Percent Root mean square Difference $(P R D)$, and the Euclidian L2 Norm standard $(L 2 N)$. Results reveal that there is not a single combination of filtering thresholds (function and value) to minimize all types of noise and interference present in $E C G$ signals. Reason why an $E C G$ denoising algorithm is proposed which allows choosing the appropriate combination (function-value) threshold, where the SNR values were the maximum and the error values were the minimum.
\end{abstract}

Keywords: $E C G$ signal, denoising, $D W T$, filtering threshold.

\section{RESUMEN}

La señal del electrocardiograma (ECG) es una bio-señal usada para determinar la salud cardiaca. Sin embargo, diferentes tipos de ruidos que acompañan a estas señales pueden esconder valiosa información para el diagnóstico de desórdenes cardiacos. Este artículo presenta un estudio experimental para remover el ruido en señales ECG usando la teoría de la Transformada Discreta de Wavelet y un set de umbrales de filtro para un eficiente filtrado. Para valorar el proceso, usamos los registros de la base de datos de arritmias del MIT-BIH (MITDB) y las señales de ruido estandarizadas (actividad muscular y contacto con el electrodo) desde la base de datos Noise Stress Test. También, a las señales ECG se les sumo señales de ruido Gaussiano blanco, presentes en señales del tipo eléctrico. Además, como primer paso consideramos la minimización de la desviación de la línea base y la interferencia de la línea de potencia. Las métricas usadas son Signal-to-Noise Ratio $(S N R)$, the Root Mean Squared Error $(R M S E)$, the Percent Root mean square Difference (PRD), and the Euclidian L2 Norm standard $(L 2 N)$. Los resultados revelan que no hay una simple combinación de umbrales de filtro (función y valor) para minimizar todos los tipos de ruido e interferencias presentes en señales ECG. Por esta razón, se propone un algoritmo de filtrado, éste permite escoger la apropiada combinación (función-valor) del umbral, donde se maximice el valor de SNR mientras que se minimicen los valores de error.

Palabras clave: señales $E C G$, filtrado, $D W T$, umbral de filtrado. 


\section{INTRODUCTION}

The electrocardiogram monitors the electrical activity of the heart. The signal is a non-stationary biosignal, and its amplitude is in the millivolts order $(1-10 \mathrm{mV})$ with an activity in a low-frequency range $(0.5-50) \mathrm{Hz}$. It is well known that $E C G$ signals are commonly accompanied by different types of noise hindering the accuracy of $E C G$ analysis (Alfaouri \& Daqrouq, 2008). Several studies presented methods for minimizing the noise in ECG signals. Sörnmo \& Laguna (2005) proposed some techniques for Power Line Interference $(P L I)$ cancellation, such as linear and nonlinear filters, estimation-subtraction techniques and band-stop filters. Singh, Kumar, \& Kumar (2014) denoised the ECG signal employing Discrete Wavelet $(D W T)$, consisting of a set of threshold filters for PLI and $B W$ interferences, and wideband stochastic noise. As metrics, they used the Mean Squared Error (MSE), SNR, and PRD, and better results were obtained applying the Daubechies and Symlet Mother Wavelet Transforms (MWT). Awal, Mostafa, Ahmad, \& Rash (2014) used the DWT and the modified S-median thresholding technique, and as metrics the MITDB (Moody \& Mark, 2001), MSE, RMSE, the improved SNR, and $P R D$. Results were good in the presence of Gaussian and color noise using a soft threshold, and results were moderate in the presence of wandering baseline line, artefact motion, and electrode movement. Overall, the $E C G$ denoising process is a complex task due to the diversity of noises present in $E C G$ signals, the low $S N R$ values, the morphological changes in the $E C G$ signals, different types of arrhythmias and the measurement in medical emergency conditions (Alfaouri \& Daqrouq, 2008; Tompkins, 2000).

This work presents an experimental study on ECG signal denoising using the MITDB from Physionet (Goldberger et al., 2000). The filtering threshold is composed by a function and a value (Donoho, 1995). A set of experiments was conducted using a DWT with the MWTs (Daubechies and Symlet wavelets), a set of standard filtering threshold values (see Table 1), and threshold functions (see Table2). To the $E C G$ records we added the noises muscles activity (" $m a$ ") and electrode-skin contact ("em") from the NSTDB (Moody \& Mark, 2001), and the additive white Gaussian noise ("wn"), using appropriate software. SNR, MSE, RMSE, PRD and the Euclidian norm, also known as the L2Norm, were used as metrics. In the Materials and Method Section we presented the database, the Wavelet theory, the threshold functions and values, while the results and their performance using threshold benchmarks are presented and discussed in the Results and Discussions Section. Finally, the main findings are summarized in the Conclusions Section.

\section{MATERIALS AND METHODS}

\subsection{Databases}

From MITDB, 10 ECG signals were analyzed in the experimental phase, namely 100, 102, 103, 105, $106,117,118,121,123$ and 202 . Their main characteristics are respectively a duration of 30 minutes, a sampling frequency of $360 \mathrm{~Hz}$, an 11 bits resolution and a 680,000 samples length. In all cases the signals were modified with three types of noise, " $m a$ ", "em" and " $w n$ ", at $S N R=10 \mathrm{~dB}$. Figure 1 shows the original ECG103 signal and the noise signals " $m a$ ", "em" and "wn", while Figure 2 depicts the $E C G 103$ record with the three types of noises and their visual effects of signal deformation over the ECG signal. 


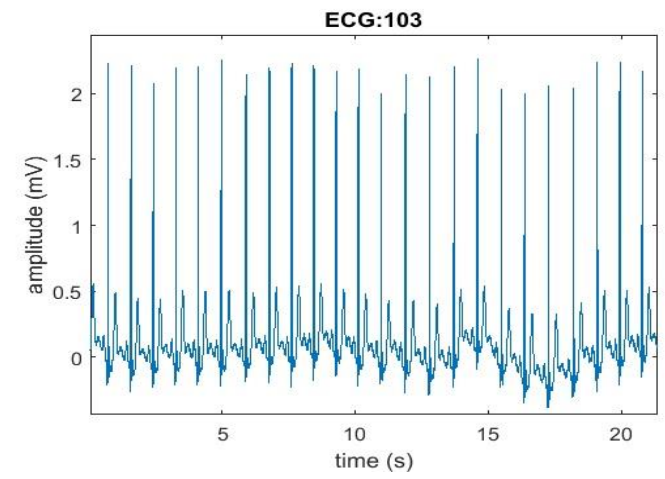

(a)

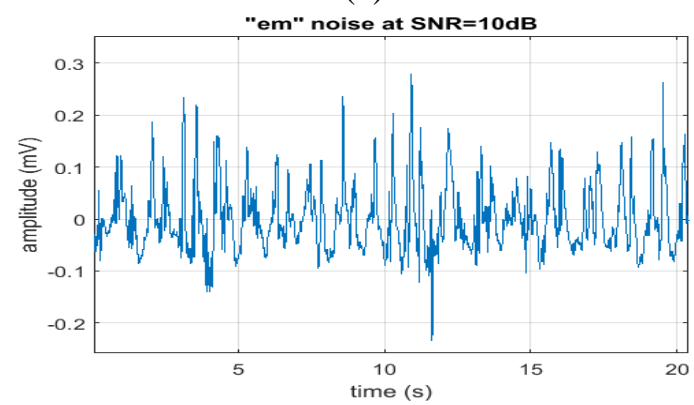

(c)

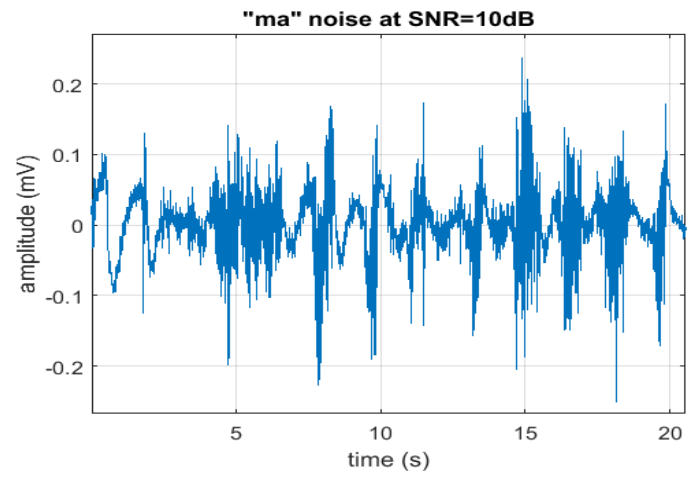

(b)

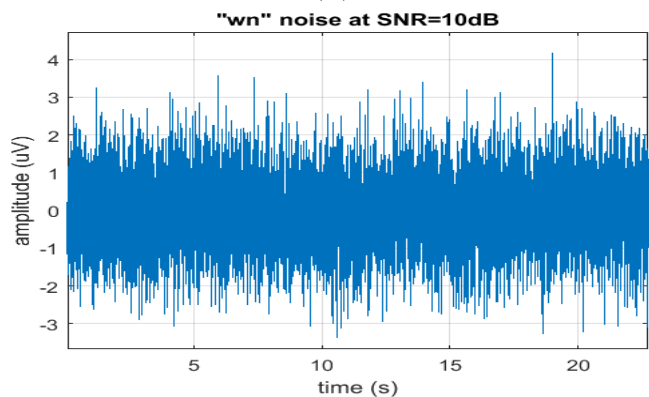

(d)

Figure 1. ECG original and noise signals: (a) ECG103, (b) " $m a$ ", (c) " $\mathrm{em}$ ", and (d) "wn".

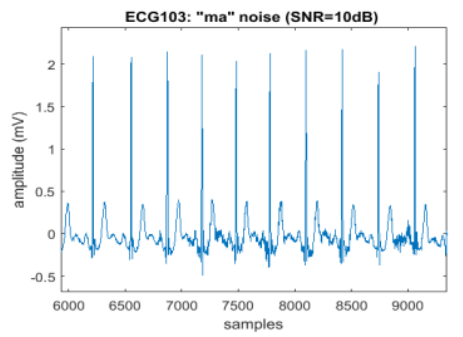

(a)

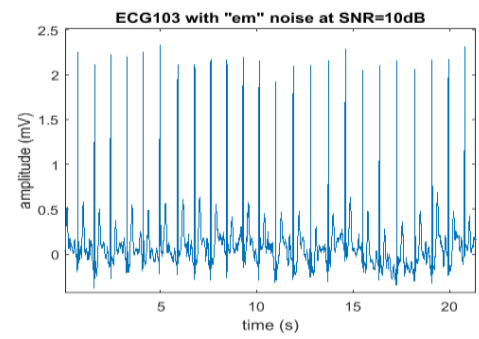

(b)

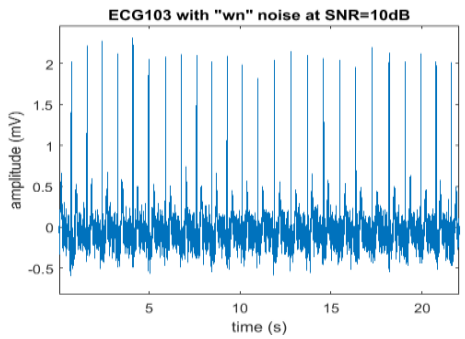

(c)

Figure 2. ECG103 record segment with considered noises at a SNR of $10 \mathrm{~dB}$ : (a) " $m a$ ", (b) "em", and (c) "wn".

\subsection{Discrete wavelet transform (DWT)}

$D W T$ works with discrete signals defined as:

$$
\operatorname{DWT}(s, \tau)=2^{\frac{-s}{2}} \sum x[n] \Psi^{*}\left(2^{-s} n-\tau\right)
$$

where, $x[\mathrm{n}]$ is the discrete time signal, $\Psi^{*}$ is the complex conjugate of the analyzing wavelet function ( $\Psi[\mathrm{n}]), s$ and $\tau$ are the dilation and location parameters respectively (Addison, 2005).

$D W T$ uses two filters, a low $(L P F)$ and a high pass filter $(H P F)$ to decompose the signal into different scales. The $L P F$ outputs are called approximation coefficients $(c A)$ and the $H P F$ outputs are named detail coefficients $(c D)$. Figure 3 a depicts the ECG103 record decomposition in $L P F$ and Figure $3 \mathrm{~b}$ depicts the HPF filters by using $D W T$ Symlet at fifth level. The $E C G$ signal sampling frequency from $M I T D B$ signals is $360 \mathrm{~Hz}$, and Figure $3 \mathrm{a}$ exhibits the 0-180, 0-90, 0-45, 0-22.5 and 0-11.25 ranges in $\mathrm{Hz}$ for the 1st, 2nd, 3th, 4th and 5th component, corresponding to low frequencies decomposition. Figure $3 \mathrm{~b}$ exhibits the 180-360, 90-180, 45-90, 22.5-45 and 11.25-22.5 ranges in $\mathrm{Hz}$ for the 1st, 2nd, 3rd, 4th and 5 th components, corresponding to high frequencies decomposition. 


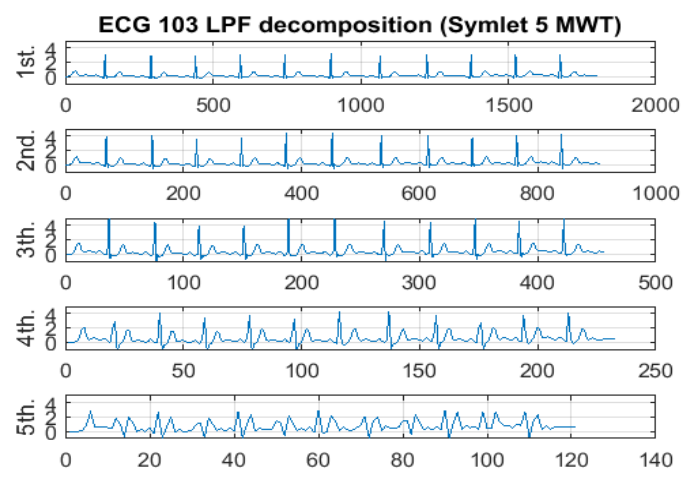

(a)

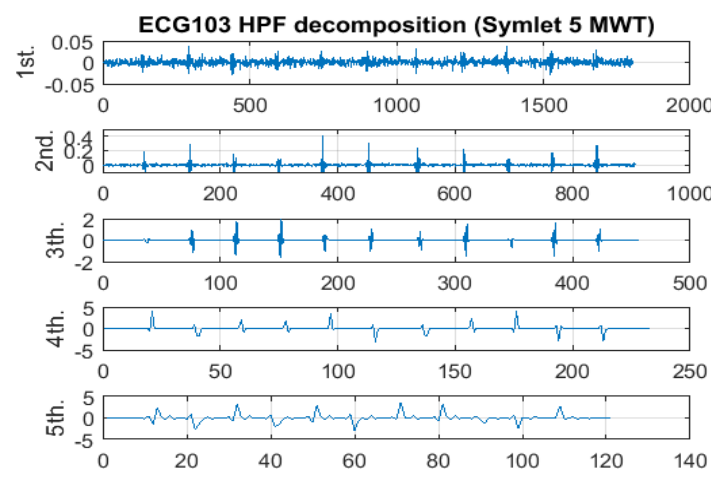

(b)

Figure 3. Decomposition of the ECG103 signal using DWT Symlet 5 at $5^{\text {th }}$ level: (a) $L P F$, decomposition levels 1, 2, 3, 4 and 5, and (b) $H P F$, decomposition levels 1, 2, 3, 4 and 5.

The inverse discrete wavelet transform $(I D W T)$ is given by:

$$
\operatorname{IDWT}(s, \tau)=A \sum \sum D W T(s, \tau) \Psi^{*}\left(2^{-s} n-\tau\right)
$$

where $A$ is a constant that do not depend of $\mathbf{x}[\mathrm{n}]$, and IDWT is the reconstructed signal (Addison, 2005).

\subsection{Wavelet threshold theory}

Wavelet denoising involves threshold filtering in which coefficients below a specific threshold value are set to zero. Two aspects must be considered, the threshold value $(\lambda)$ and the threshold function $(W j)$. Table 1 shows some threshold functions, where $W j$ are the coefficients at decomposition $\mathrm{j}$ level; the $\alpha$ value is a real number which can be adjusted freely and depend on the results reached on the denoising process. Table 2 shows some of the threshold values $(\lambda)$, where $N$ is the signal length and some parameters are in the characteristics column. For the s-median threshold value, the parameter $b$ value is defined by a set of values that vary according to the noise to be minimized $(-113,-120,-122$ and -86 for "wn", " $m a$ ", "em" and composite noises respectively; Awal et al., 2014).

Table 1. Wavelet threshold functions $(W j)$.

\begin{tabular}{lll}
\hline Function & Formula & Condition \\
\hline Hard (Donoho, 1995) & $W j=W j$ & if $|W j| \geq \lambda$ \\
& $W j=0$ & if $|W j|<\lambda$ \\
\hline Soft (Donoho, 1995) & $W j=\operatorname{sgn}(W j)(|W j-\lambda|)$ & if $|W j| \geq \lambda$ \\
& $W j=0$ & if $|W j|<\lambda$ \\
\hline Garrote (Jing-yi et al., 2016) & $W j=W j-\left(\lambda^{2} / W j\right)$ & if $|W j| \geq \lambda$ \\
& $W j=0$ & if $|W j|<\lambda$ \\
\hline Semisoft (Jing-yi et al., 2016) & $W j=\operatorname{sgn}(W j)(|W j-\mathrm{T} \lambda|)$ & if $|W j| \geq \lambda$ \\
& $W j=0$ & if $|W j|<\lambda$ \\
\hline Neighboring (Singh et al., & $W j=W j\left(1-\left(\lambda^{2} / W j^{2}\right)\right)$ & if $|W j| \geq \lambda$ \\
2014) & $W j=0$ & if $|W j|<\lambda$ \\
\hline Jing (Jing-yi et al., 2016) & $W j=\operatorname{sgn}(W j)\left(|W j|-\lambda /\left(\exp ^{3}[\alpha(|W j|-\lambda)] / \lambda\right)\right.$ & if $|W j| \geq \lambda$ \\
& $W j=0$ & if $|W j|<\lambda$ \\
\hline
\end{tabular}

\subsection{ECG denoising scheme}

Figure 4 shows the general scheme of the analysis, in which it is shown that the ECG signals from $M I T D B$ pass through a preprocessing stage (PLI and $B W$ minimization). The noises " $m a$ ", "em" and "wn" were added to partial ECG records. Then, DWT transformed the ECG signal into components 
being the result of passing the signal through respectively low and high pass filters in a decomposition stage. Thereafter, we selected the $M W T$ and the depth level we want to arrive to minimize the noise frequencies. Ultimately, we tested the different threshold sets (function and values), and finally, the $I D W T$ (re-composition stage) was applied to rebuild the total ECG signal.

Table 2. Wavelet threshold values $(\lambda \mathrm{j})$.

\begin{tabular}{lll}
\hline Function & Formula & Features \\
\hline Sqtwolog (Donoho, 1995) & $\lambda j=\sigma j \sqrt{2 \log (N j)}$ & $\sigma j=$ median $(|W j|) / 06745$ \\
\hline Minimaxi (Donoho, 1995) & $\begin{array}{l}\lambda j=\sigma j(0.39+0.19 \log 2 N j) \\
\lambda j=0 \text { if } N j<32\end{array}$ & $\sigma j=$ median $(|W j|) / 06745$ \\
\hline $\begin{array}{ll}\text { Georgeiva (Georgeiva } \text { et al., } \\
\text { 2016) }\end{array}$ & $\lambda j=\sigma j \sqrt{2 \log (N j)} / \mu j$ & $\mu=\max (|\mathrm{Wj}|)$ \\
\hline Alfauri (Alfauri et al., 2016) & $\lambda j=\mathrm{C} \sqrt{\frac{\sigma(X(n)) N j}{\sigma j}}$ & $\begin{array}{l}\mathrm{C}=5 \\
\mathbf{x}(\mathrm{n}) \text { noise signal }\end{array}$ \\
\hline S-Median (Awal et al., 2014) & $\lambda j=\frac{\sigma j \sqrt{2 \log (N j)}}{S L K+b}$ & $\begin{array}{l}\mathrm{B} \rightarrow \text { tuning factor } \\
\mathrm{L} \rightarrow \text { deepest level } \\
\mathrm{K} \rightarrow \text { level of } \lambda\end{array}$ \\
& where $S L K=2^{L-(K / L)}$ &
\end{tabular}

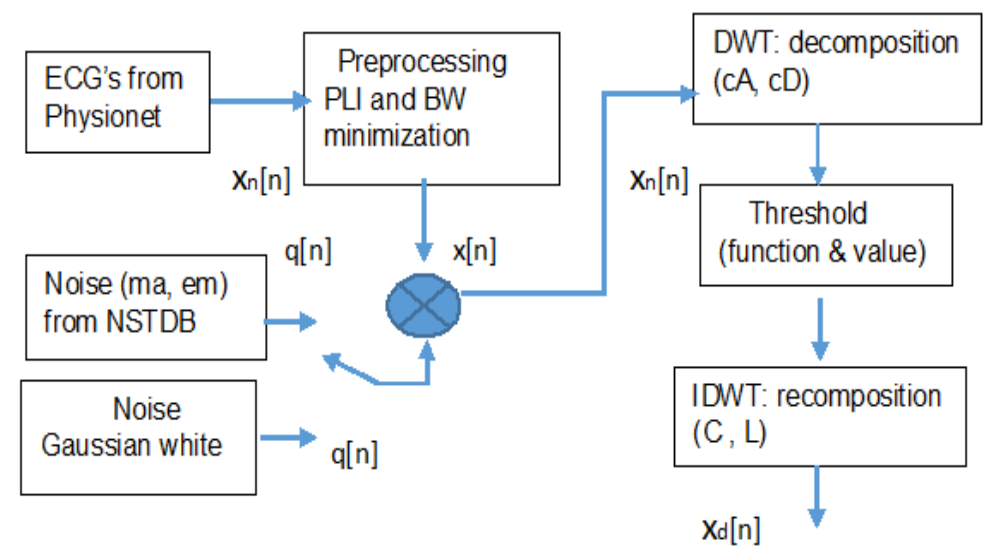

Figure 4. $E C G$ denoising scheme for experimental study of threshold values and functions.

From the general scheme, we know the original signal $\mathbf{x}[n]$ and the noise signal $\mathbf{q}[\mathrm{n}]$, variables expressed by the next expression.

$$
\mathbf{x}_{\mathrm{n}}[\mathrm{n}]=\mathbf{x}[\mathrm{n}]+\mathbf{q}[\mathrm{n}]
$$

where $\boldsymbol{x}_{\mathrm{n}}[\mathrm{n}]$ is the noise signal. Considering these variables, Table 3 shows the metric parameters used to measure the quality of the noise reduction.

Table 3. Metric parameters.

\begin{tabular}{ll}
\hline Metrics & Formula \\
\hline$S N R$ & $10 \log _{10} \frac{\sum_{n=1}^{N-1} x[n]^{2}}{\sum_{n=1}^{N-1}(x[n]-x d[n])^{2}}$ \\
\hline$M S E$ & $1 / \mathrm{N}\left(\sum_{n=1}^{N-1}(\boldsymbol{x}[n]-\boldsymbol{x d}[n])^{2}\right)$ \\
\hline$R M S E$ & $\sqrt{\left.1 / \mathrm{N}\left(\sum_{n=1}^{N-1}(\boldsymbol{x}[n]-\boldsymbol{x d} d n]\right)^{2}\right)}$ \\
\hline
\end{tabular}




\begin{tabular}{ll}
\hline PRD & $\sqrt{\frac{\sum_{n=1}^{N-1}(\boldsymbol{x}[n]-\boldsymbol{x d}[n])^{2}}{\sum_{n=1}^{N-1} x[n]^{2}}} * 100$ \\
\hline NormL2 & $\sqrt{\sum_{n=1}^{N-1} x[n]^{2}}$ \\
\hline
\end{tabular}

where $\mathbf{x}_{\mathbf{d}}[\mathrm{n}]$ is the signal without noise.

\subsection{Denoising process}

Generally, the most common sources of noise are $P L I, B W$ interference, motion artifacts, electrical and muscles activity (" $m a$ "); instability of electrode-skin contact (" $\mathrm{em}$ ") and white noise ("wn") (Tompkins, 2000; Georgieva \& Tcheshmedjiev 2013). In our analysis noise was treated separately for PLI and $B W$ as extrinsic noises, and the " $m a$ ", "em" and " $w n$ " noises were treated as intrinsic noise. Table 4 shows the ECG proposed signal denoising algorithms.

Table 4. ECG signal denoising process algorithm

\begin{tabular}{|c|c|}
\hline Step & Description \\
\hline 1 & The $E C G$ signals are obtained from $M I T D B$ \\
\hline 2 & $\begin{array}{l}P L I \text { Identification (by using Fast Fourier Transform, } F F T \text { ), } B W \text { interference identification } \\
\text { by base line wandering. The " } m a \text { ", "em" and " } w n \text { " identified as an } E C G \text { signal with } \\
\text { distortions around its characteristics (see Fig. 1). }\end{array}$ \\
\hline 3 & $\begin{array}{l}\text { Apply the } D W T \text { and a selected } M W T \text { at corresponding level, to obtain the } c A \text { and } c D \\
\text { coefficients. For } P L I \text { we minimized the } c D \text { coefficients at } 2^{\text {nd }} \text { level. For } B W \text { we minimized } \\
\text { the } c A \text { coefficient at } 8^{\text {th }} \text { level. For the intrinsic noise, we minimized the } c A \text { coefficients at } \\
7^{\text {th }} \text { and } 8^{\text {th }} \text { levels and the } c D \text { coefficients at all levels. }\end{array}$ \\
\hline 4 & Use a set of thresholds (function and value) to obtain the filtering coefficient. \\
\hline 5 & Use the $I D W T$ to obtain the denoised signal $\mathbf{x}_{\mathrm{d}}[\mathrm{n}]$. \\
\hline 6 & Apply the metrics to find results. \\
\hline 7 & Back to step 4 to select a new filter; repeat this until to complete the threshold set. \\
\hline 8 & Back to step 3 to select a new $M W T$, repeat this until completing the $M W T$ set. \\
\hline 9 & Tabulate the data. \\
\hline 10 & Choose the better results. \\
\hline
\end{tabular}

\section{RESULTS AND DISCUSSION}

\subsection{PLI denoising}

Figure 5 shows the $P L I$ minimization stage (PLI marked in red ellipse, middle signal), and its minimizing results (marked in red ellipse, lower signal). The best result obtained, was applying Symlet 8 at $2^{\text {nd }}$ level $(M W T)$ and the process suggested in Table 4. Figure 6 shows a benchmarking between the threshold (value/function) set, sqtwolog/hard (sqt/h); minimaxi/hard $(\mathrm{min} / \mathrm{h}) ;$ Georgeiva/hard $(\mathrm{Geo} / \mathrm{h})$ and sqtwolog/semisoft (sqt/semi). The results show that the threshold pair $(\mathrm{min} / \mathrm{h})$ has the higher $S N R$ value (Figure 6a). Figure 6b depicts the lowest $P R D$ values for all $E C G$ records $(100,102,103,105,106$ and 202), revealing the existence of a minimum difference between $\mathrm{sqt} / \mathrm{h}$ and $\mathrm{min} / \mathrm{h}$ threshold combinations. Before applying the PLI minimization method it is necessary to verify the existence of the PLI interference, because some $E C G$ records as 117, 118, 119, 122 and 123 did not present this interference. 

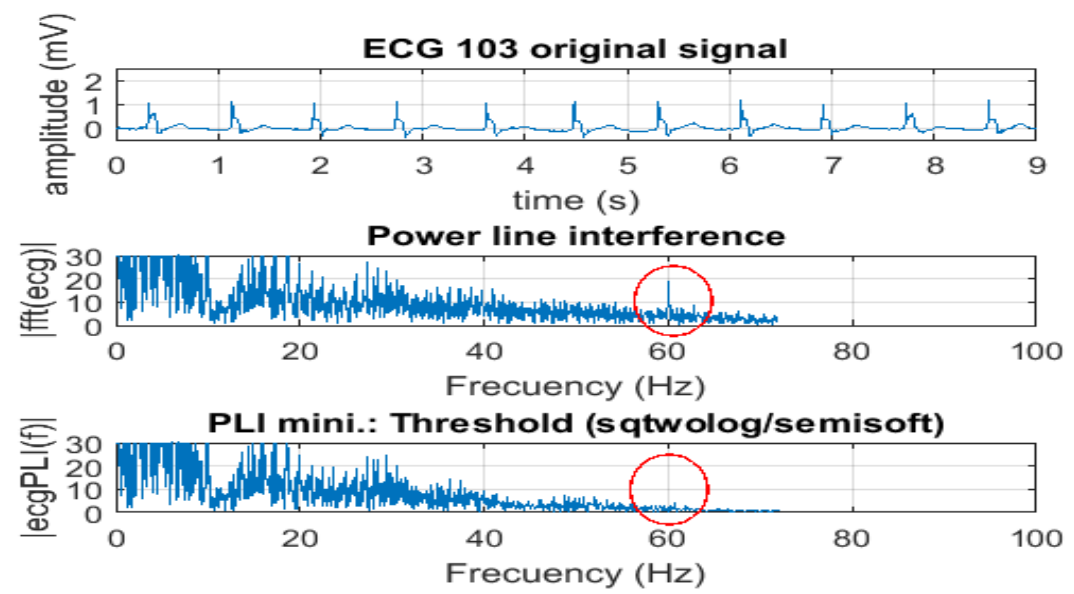

Figure 5. $P L I$ denoising for ECG103 record: (a) original signal (on top), $P L I$ identification by using Fast Fourier Transform over the signal (signal at $60 \mathrm{~Hz}$, red ellipse) (in middle), and the PLI minimization (signal minimization, red ellipse) (on bottom).

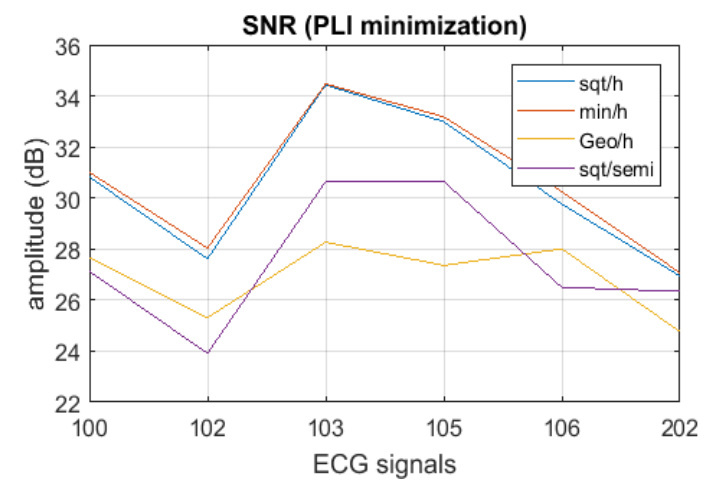

(a)

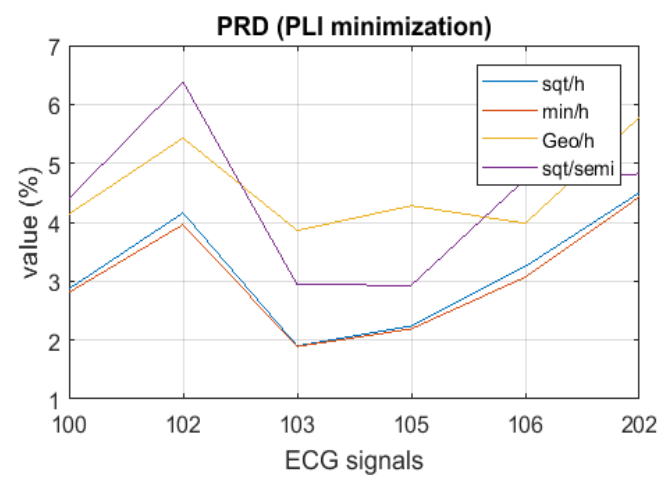

(b)

Figure 6. Benchmarking between thresholds (values/functions) for PLI minimization, (a) $S N R$, (b) PRD.

\subsection{BW minimization}

$B W$ interference is a low frequency signal, less than $1 \mathrm{~Hz}$ (Sörnmo \& Laguna, 2005). Figure 7 shows the baseline wandering (red line) over the ECG103 register. We experimented with Daubechies and Symlet, mother wavelets. The best results were obtained was using the Symlet 8 at $8^{\text {th }}$ level and the process suggested in Table 4.

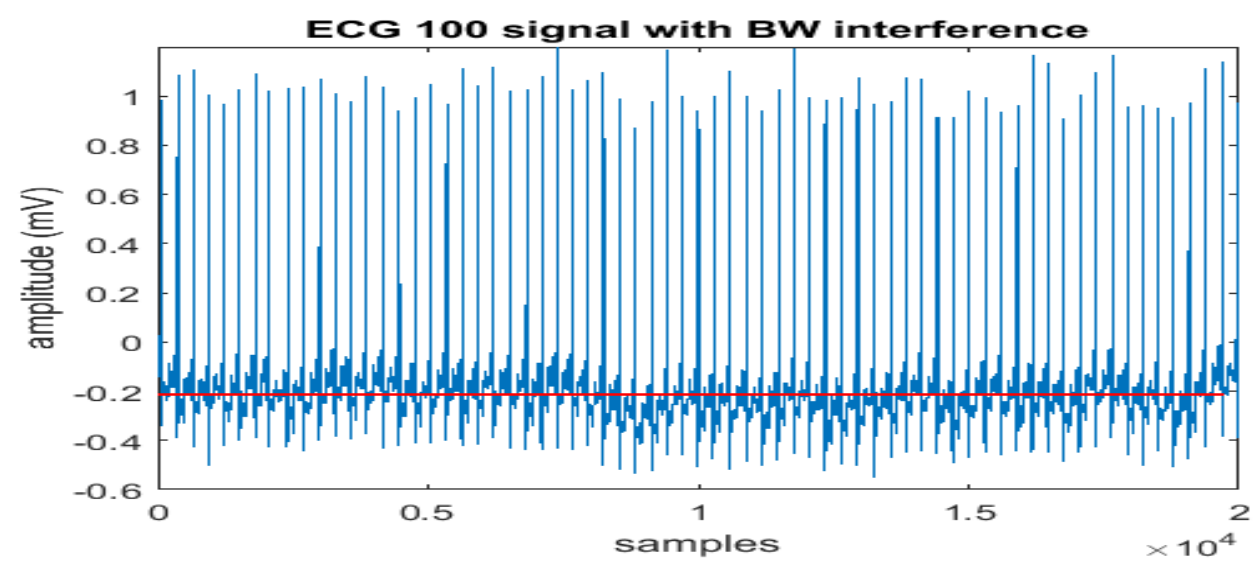

Figure 7. $E C G 103$ record with $B W$ interference. 
Figure 8a shows the $B W$ minimization for the ECG103 record considering the use of a threshold set. The best result was obtained using the sqtwolog/soft combination (top figure); the red ellipse over the $E C G$ signals shows the $B W$ presence when we used the other threshold combinations. Figure $8 \mathrm{~b}$ shows the benchmarking process for $B W$ minimization of $E C G 100,102,103,105,106,202,117,118$ 119,122 and 123 records (x axis). The $L 2 N$ was respectively determined for the original signal and for the denoised signal obtained after application of the threshold combination. When the threshold with sqtwolog value and soft function were used (minimum distance, red signal) yielded the best results.

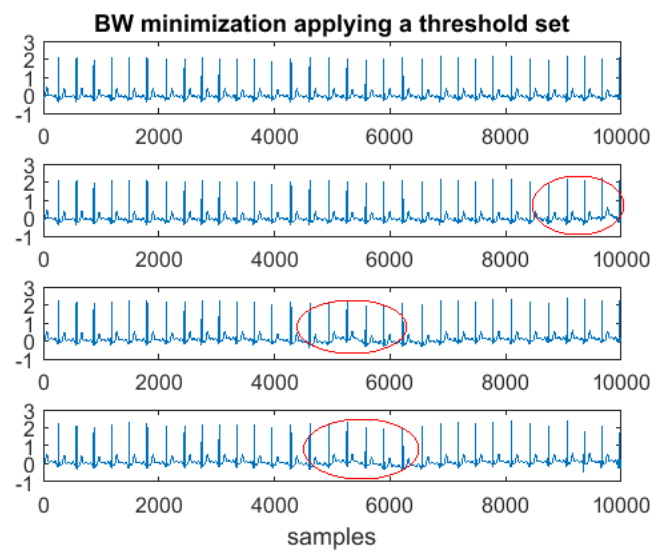

(a)

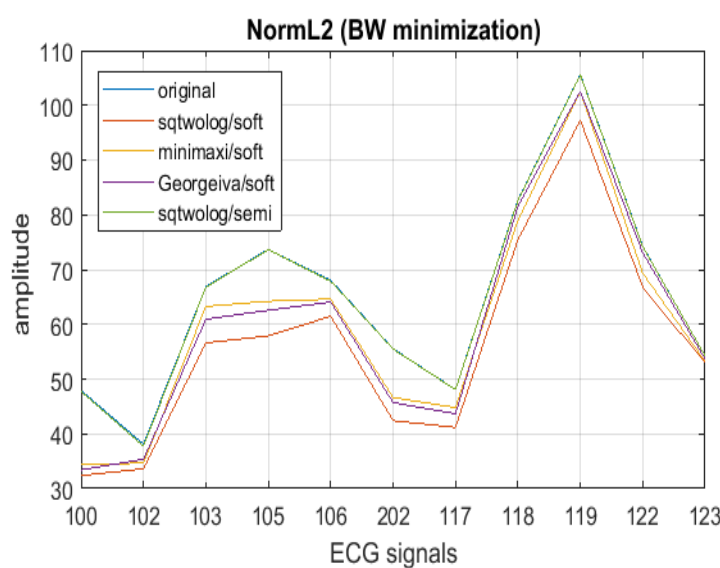

(b)

Figure 8. ECG103 $B W$ minimization by using a threshold set, from top to bottom: sqtwolog/soft, minimaxi/soft, Georgeiva/soft and sqtwolog/semisoft (red ellipse shows $B W$ interference).

\subsection{Intrinsic noise minimization}

Figure 9a shows the ECG103 denoising results when we added $m a$ noise at $S N R=10 \mathrm{~dB}$. We can see the signal morphology comparison between the denoising signals, where the best result was for the sqtwolog/hard threshold. These results in the bar Figure 9b shows the benchmarking where the $S N R$ (highest), RMSE and PRD (lower) values when we used the sqtwolog/hard threshold.

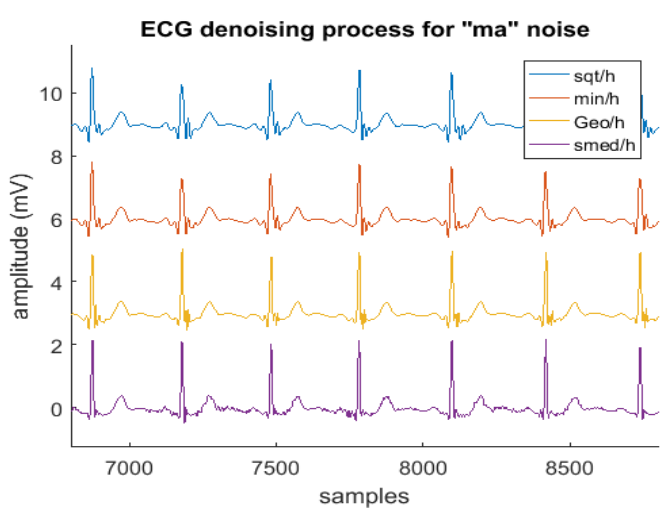

(a)

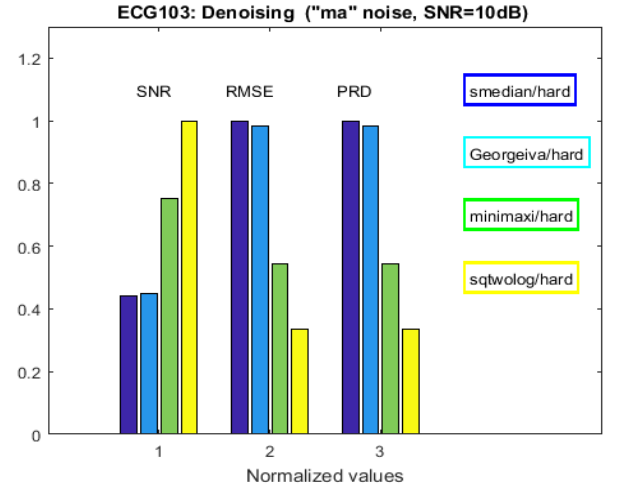

(b)

Figure 9. ECG103 ma denoising using a set of thresholds: (a) signal comparison; and (b) metric benchmarking (normalized values).

Figure 10a shows the ECG103 denoising results when the em noise was added at $S N R=10 \mathrm{~dB}$. This figure enables comparison of the signal morphology, and at naked eyes no substantial changes are observable. In the bar chart (Figure 10b), the benchmarking shows the SNR (heighted value) and the RMSE and PRD (lower values) for sqtwolog/hard threshold combination. For smedian/hard 
combination, the morphology signal appears clearer. In generally terms we could said that all threshold combinations could help the process of removing this type of noise.

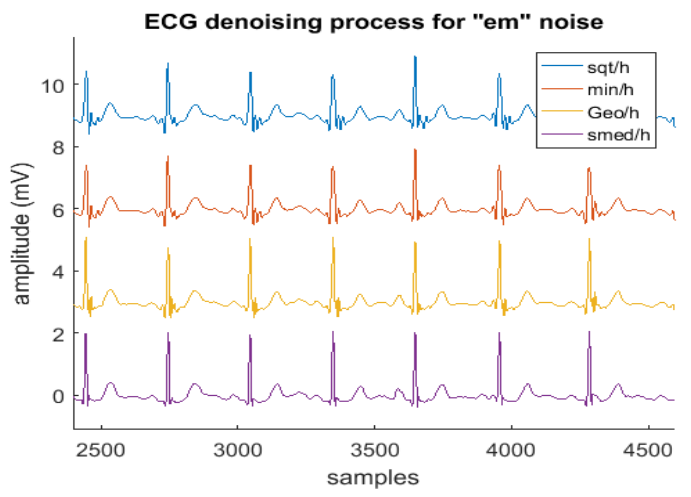

(a)

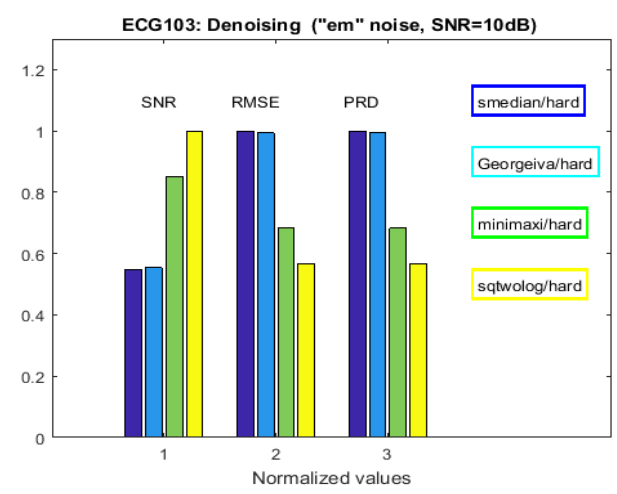

(b)

Figure 10. ECG103 em denoising using a set of thresholds: (a) signal comparison; (b) metric benchmarking.

Figure 11a shows the $E C G 103$ denoising results for $w n$ noise at $S N R=10 \mathrm{~dB}$. We can see the signal morphology comparison, where the first three combination methods (sqtwolog/hard, minimaxi/hard and Georgeiva/hard) could favorably assist in trimming noises. In the bar chart (Figure 11b), the benchmarking shows the $S N R$ (highest values) for minimaxi/hard combination, and the $R M S E$ and $P R D$ (lower values) for sqtwolog/hard combination, and according the morphology of the signal the Georgeiva/hard combination give a clear signal. We could conclude that the three before mentioned approaches could be used to remove or minimized the considered types of noise.

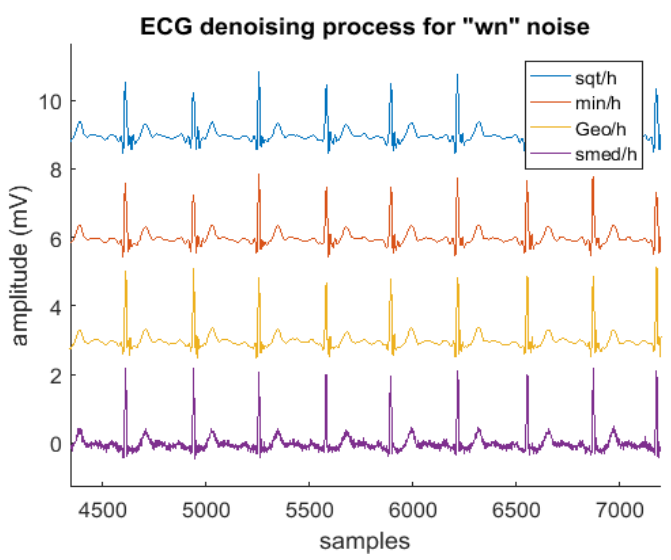

(a)

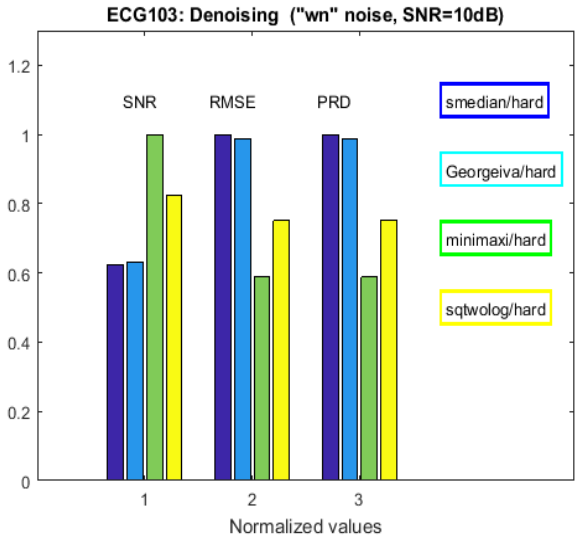

(b)

Figure 11. ECG103 wn denoising: (a) signal comparison; (b) metric benchmarking.

\section{CONCLUSIONS}

The experimental study presented herein enables choosing the best combination of threshold values and threshold functions from $D W T$ theory for the noise reduction of $E C G$ signals using a proposed algorithm. We used ten $E C G$ signals obtained from the $M I T D B$ and the noises from NSTDB, both database from Physionet. The method used treated the denoising process according to the noise characteristics. Extrinsic interferences, as $P L I$, were minimized using Symlet 8 at the $8^{\text {th }}$ level with a threshold using sqtwolog / hard combination (value and function), where the detail coefficients of the second level were minimized. $B W$ was minimized using Symlet 8 at $8^{\text {th }}$ level with a threshold using sqtwolog / hard combination, where the approximation coefficients at the eighth level were minimized. For intrinsic noise minimization, the best results were obtained when we used the same mother wavelet, with a 
threshold using sqtwolog / hard combination for " $m a$ " and "em" noises, and minimaxi / hard combination for " $w n$ " noise, where all detail coefficients and the two last approximation coefficients were minimized. As conclusion, we can say that for $E C G$ signals, with intrinsic and extrinsic noises, the best way using the wavelet transform theory is to have a tool that permits to choose the adequate threshold combination (function \& value).

\section{REFERENCES}

Addison, P. S. (2005). Wavelet transforms and the ECG: a review. Physiological Measurement, 26(5), 155-199. https://doi.org/10.1088/0967-3334/26/5/R01

Alfaouri, M., Daqrouq, K. (2008). ECG signal denoising wavelet transform thresholding. American Journal of Applied Sciences, 5(3), 276-281.

Awal, A., Mostafa, S., Ahmad, M., Rash, M. (2014). An adaptive level dependent wavelet thresholding for ECG denoising. Biocybernetic and Biomedical Engineering, 34(4), 238-249. https://doi.org/10.1016/j.bbe.2014.03.002

Donoho, D. (1995). De-noising by soft-thresholding. IEEE Transctions on Information Theory, 41, 613-627.

Goldberger, A., Amaral, L., Glass L, Hausdorff, J., Ivanov, P., Mark, R., Mietus, J., Moody, G., Peng, C., Stanley, H. (2000). PhysioBank, PhysioToolkit, and PhysioNet: Components of a new research resource for complex physiologic signals. Circulation, 101(23), e215-e220. https://doi.org/10.1161/01.CIR.101.23.e215

Georgieva, G., Tcheshmedjiev, K. (2013). Denoising of electrocardiogram data with methods of wavelet transform. International conference on computer system and technologies CompSysTech'13, University of Ruse, pp. 9-19.

Jing-yi, L., Hong, L., Dong, Y., Yan-sheng, Z. (2016). A new wavelet threshold function and denoising application. Mathematical Problems in Engineering, 2016, 8 p. http://dx.doi.org/10.1155/2016/3195492

Moody, G. B., Mark, R. G. (2001). The impact of the MIT-BIH Arrhythmia Database. IEEE Engineering in Medicine and Biology Magazine, 20(3), 45-50. https://doi.org/10.1109/51.932724

Singh, M., Kumar, R., Kumar, A. (2014). Comparison between different wavelet transforms and thresholding techniques for ECG denoising. IEEE International Conference on Advances in Engineering and Technology Research, Unnao, India, 6 p. htpps://doi.org/ 10.1109/ICAETR.2014.7012899

Sörnmo, L., Laguna, P. (2005). Bioelectrical signal processing in cardiac and neurological applications. Oxford, UK: Academic Press. 688 p.

Tompkins, W. J. (2000). Biomedical digital signal processing. New Jersey, US: Prentice Hall. 378 p. 